\title{
Total extraperitoneal endoscopic hernioplasty (TEP) versus Lichtenstein hernioplasty: a systematic review by updated traditional and cumulative meta-analysis of randomised-controlled trials
}

\author{
P. Gavriilidis ${ }^{1} \cdot$ R. J. Davies ${ }^{2} \cdot$ J. Wheeler $^{2} \cdot$ N. de'Angelis ${ }^{3} \cdot$ S. Di Saverio ${ }^{2}$ (D)
}

Received: 4 July 2019 / Accepted: 4 September 2019 / Published online: 10 October 2019

(c) The Author(s) 2019

\begin{abstract}
Background-purpose Totally extraperitoneal (TEP) endoscopic hernioplasty and Lichtenstein hernioplasty are the most commonly used approaches for inguinal hernia repair. However, current evidence on which is the preferred approach is inconclusive. This updated meta-analysis was conducted to track the accumulation of evidence over time.

Methods Studies were identified by a systematic literature search of the EMBASE, PubMed, Cochrane Library, and Google Scholar databases. Fixed- and random-effects models were used to cumulatively assess the accumulation of evidence over time.

Results The TEP cohort showed significantly higher rates of recurrences and vascular injuries compared to the Lichtenstein cohort; [Peto Odds ratio $(\mathrm{OR})=1.58(1.22,2.04), p=0.005$ ], [Peto $\mathrm{OR}=2.49(1.05,5.88), p=0.04]$, respectively. In contrast, haematoma formation rate, time to return to usual activities, and local paraesthesia were significantly lower in the TEP cohort compared to the Lichtenstein cohort; [Peto OR $=0.26(0.16,0.41), p \leq 0.001$ ], [mean difference $=-6.32(-8.17,-4.48)$, $p \leq 0.001]$, [Peto $\mathrm{OR}=0.26(0.17,0.40), p \leq 0.001]$, respectively.

Conclusions This study, which is based on randomised-controlled trials (RCTs) of high quality, showed significantly higher rates of recurrences and vascular injuries in the TEP cohort than in the Lichtenstein cohort. In contrast, rate of postoperative haematoma formation, local paraesthesia, and time to return to usual activities were significantly lower in the TEP cohort than in the Lichtenstein cohort. Future multicentre RCTs with strict adherence to the standards recommended in the Consolidated Standards of Reporting Trials guidelines will shed further light on the topic.
\end{abstract}

Keywords Total extraperitoneal hernioplasty $\cdot$ TEPP $\cdot$ Lichtenstein technique $\cdot$ Inguinal hernia repair $\cdot$ Mesh $\cdot$ Hernia repair · Groin hernia

Electronic supplementary material The online version of this article (https://doi.org/10.1007/s10029-019-02049-w) contains supplementary material, which is available to authorized users.

\section{S. Di Saverio}

salo75@inwind.it; salomone.disaverio@gmail.com; salomone.disaverio@addenbrookes.nhs.uk

1 Division of Gastrointestinal and Hepato-Biliary-Pancreatic Surgery, Imperial College Healthcare NHS Trust, Hammersmith Hospital, London W12 0HS, UK

2 Cambridge Colorectal Unit, Addenbrooke's Hospital, Cambridge University Hospitals NHS Foundation Trust, Hills Road, Cambridge CB2 0QQ, UK

3 Department of Digestive Surgery, Henri Mondor University Hospital, 94010 Créteil, France

\section{Introduction}

Inguinal hernia repair is the most common operation in general surgery with more than 20 million performed annually worldwide [1]. Most patients with an inguinal hernia are symptomatic and the treatment of choice is surgical repair with mesh using open or laparo-endoscopic approach. The use of mesh varies worldwide from 0 to $5 \%$ in low-resource countries to $95 \%$ in high-resource countries. The Swedish National registry reported that for the year 2015, the percentages of inguinal hernia repair techniques were as follows: Lichtenstein hernioplasty 64\%, totally extraperitoneal (TEP) hernioplasty $25 \%$, transabdominal preperitoneal (TAPP) hernioplasty $3 \%$, open preperitoneal hernioplasty $3.3 \%$, and 
tissue repair $0.8 \%$. The German Herniamed registry reported the following data for the period from 2009 to 2016: TAPP hernioplasty $39 \%$, TEP hernioplasty $25 \%$, and Lichtenstein hernioplasty $24 \%$. There is a lack of data from America and Asia [1].

Some possible complications of hernioplasty include recurrence necessitating reoperations in $10-15 \%$ of cases and chronic pain (lasting more than 3 months) in $10-12 \%$ of cases, which may lead to long-term disability [1].

To date, the evidence comparing TEP hernioplasty to Lichtenstein hernioplasty is non-conclusive [2, 3]. However, there has been new published evidence since the most recent meta-analysis. Therefore, we decided to perform an updated traditional and cumulative meta-analysis to estimate the impact of the new studies on the robustness of the statistical significance of existing meta-analyses comparing TEP hernioplasty and Lichtenstein hernioplasty. Recurrence rate and chronic persistent pain were selected as primary outcomes.

\section{Methods}

The preferred reporting items for systematic reviews and meta-analyses' checklist was followed in this study [4].

\section{Literature search}

With the use of the search terms in the free text and Medical Subject Headings terms ("laparoscopic or endoscopic total extraperitoneal inguinal repair", "laparoscopic or endoscopic total extraperitoneal inguinal hernioplasty", "open with mesh Lichtenstein inguinal hernia repair", "Lichtenstein's technique", "inguinal hernia repair with mesh", "TEP", "inguinal hernia", or "randomised or randomized controlled trial"), a systematic search of literature published over the last 30 years was performed using the EMBASE, Medline (PubMed), Cochrane Library, and Google Scholar databases. A grey literature search was also performed in the clinicaltrials.gov website. References of the retrieved articles were checked manually for additional studies. Disagreements between the authors were resolved by consensusbased discussions.

\section{Study selection, and inclusion and exclusion criteria}

Only randomised-controlled trials (RCTs) that compared TEP laparoscopic inguinal hernia repair with Lichtenstein's technique for inguinal hernia repair were included in this study. They fulfilled the following criteria: (1) clearly documented comparison of TEP laparoscopic approach and Lichtenstein's technique for inguinal hernia repair, (2) report of at least one outcome measure, (3) inclusion of only the most recent publication in cases of multiple publications by the same institution, and (4) selection of TEP and Lichtenstein approaches from multi-arm RCTs.

Abstracts, retrospective studies, and non-English language publications were excluded from the analysis.

\section{Data extraction and outcomes}

Two reviewers (PG and NA) independently extracted the following summary data from the included studies: name of authors; year of publication; number of patients included in the TEP and Lichtenstein hernioplasty cohorts; duration of operation; conversion rate; rates of haematoma and seroma formation; incidences of wound infection, vascular injury, and visceral injury; time to return to usual activities; incidence of persisting pain or persisting numbness; and recurrence rate.

\section{Definitions}

Hernia recurrence was defined as any symptomatic or asymptomatic palpable lump or weakness in the operated groin found by the patient or the examining physician and exacerbated by the Valsalva manoeuvre. Chronic persisting pain was defined as pain of any severity (including testicular) persisting for more than 3 months after the operation. Impaired sensibility was defined as loss of the ability to register touch or the presence of numbness and tingling. Wound infection, vascular injury, and visceral injury were reported according to the definitions provided by the authors of the included studies. Operative time was defined as the time from the initial operative scalpel-to-skin contact to the placement of the last suture. Haematomas included wound and scrotal haematomas or ecchymoses but not bruising, and seromas included hydroceles. Time to return to usual activities was defined as the time taken to get back to normal social activities or work.

\section{Statistical analysis}

The methodological quality of all included RCTs was based on Cochrane's criteria, which include random sequence generation, allocation concealment, blinding of participants and personnel, blinding of outcome assessment, incomplete outcome data, selective reporting, and differences in baseline characteristics [5].

Statistical analysis was conducted using the STATA software (version 15, Stata Corp LP, College Station, TX, USA) and the Review Manager 5.3 software (Cochrane Collaboration, Oxford, England). Heterogeneity was assessed using the $I^{2}$ test, and cut-off values of $25 \%, 50 \%$, and $75 \%$ were considered of low, moderate, and high heterogeneity, respectively [6]. Where heterogeneity occurred, both fixed- and random-effects 
models were generated, and the conclusions compared, with the latter used where there were discrepancies. Fixed-effects models were used in cases of $I^{2}$ value less than $25 \%$.

Dichotomous variables were analysed based on odds ratios (ORs) with 95\% confidence intervals. For the outcomes considered, the reference categories were selected, such that $\mathrm{OR}<1$ TEP.

Continuous variables were combined based on the mean difference (MD) and the standardised MD. The studies were then combined using the Mantel-Haenszel method in the first instance, with the Peto approach used when the cross-table has a zero cell $[5,6]$. For studies that did not report the means and variances of the two groups, these values were estimated from the median, range, and the size of sample, using the technique described by Hozo et al. where possible [7].

In all analyses, the point estimate was considered significant at $P<0.05$.

\section{Sensitivity analysis}

Analyses of both primary and secondary outcomes were calculated using the random-effects and fixed-effect models to assess the impact of heterogeneity on the robustness of the conclusions. Cumulative analysis was performed to track the accumulation of evidence and to determine if the results of the meta-analysis were dominated by a particular study [8].

\section{Results}

\section{Search strategy and included study characteristics}

Twenty-one studies including 6573 patients were selected from a pool of 366 studies (Fig. 1). Of these patients, 3242 (49.3\%) and 3331 (50.7\%) underwent TEP and Lichtenstein hernioplasty, respectively [9-29]. Two abstracts, 1 in German and 1 in Spanish articles, were excluded. Non-significant differences were found in the demographic characteristics between the two cohorts (Table 1).

\section{Quality assessment of included RCTs}

The methodological quality of the RCTs was poor; only 4 of the 21 studies blinded participants and personnel, and one of them blinded the assessors of the outcomes (Table 2).

\section{Primary outcomes}

\section{Recurrences}

There was evidence of a higher recurrences in the TEP cohort (149/2678 patients; $6 \%$ of patients) compared with the Lichtenstein cohort (99/2790 patients; $4 \%$ of patients) [Peto OR $=1.58$ (1.22, 2.04), $p=0.005$ ] (Table 1, Fig. 2).

\section{Chronic persistent pain}

There was no difference in chronic pain between the TEP cohort (185/1617 patients; $11 \%$ of patients) and the Lichtenstein cohort (228/1862 patients; $13 \%$ of patients) [Peto $\mathrm{OR}=0.81(0.66,1.00), p=0.05]($ Table 1$)$.

\section{Statistically significant secondary outcomes}

There was evidence that the operative time was significantly shorter (by $11 \mathrm{~min}$ ) in the Lichtenstein cohort than in the TEP cohort. Vascular injuries were significantly less in the Lichtenstein cohort than in the TEP cohort (Table 1).

There was evidence that the outcomes of haematoma formation rate, return to usual activities, and local paraesthesia were significantly better in the TEP cohort than in the Lichtenstein cohort (Table 1).

\section{Statistically non-significant secondary outcomes}

Non-significant differences were observed in the outcomes of seroma formation rate, incidence of wound infections, and time to discharge (Table 1).

\section{Sensitivity analysis}

Analysis of outcomes using fixed- and random-effects models did not reveal any discrepancies. Cumulative metaanalysis further supports the evidence that the recurrence rate was significantly lower in the Lichtenstein procedure. It depicts two periods one until 2004 where the differences were non-significant and the second which starts with the study of Neumayer in 2004 until the present day where the Lichtenstein repair demonstrates a significantly lower recurrence rate. Interestingly, the high-quality RCT by Gutlic [29] did not influence the results significantly either way (Fig. 3).

\section{Discussion}

This study shows that the TEP cohort had a higher recurrence rate and vascular injury rate than the Lichtenstein cohort. However, the TEP cohort had better outcomes in terms of haematoma formation rate, time to return to usual activities, and local paraesthesia compared with the Lichtenstein cohort. No difference was found between the 2 cohorts in terms of wound infection, persistent pain, and time to discharge.

The recurrence rate is difficult to explore, because it depends on varied follow-up periods [30]. The followup periods in this study also varied widely. Usually, the recurrence rate is estimated as twice the number of reoperations [31]. A Danish observational study reported that 


\section{PRISMA 2009 Flow Diagram}
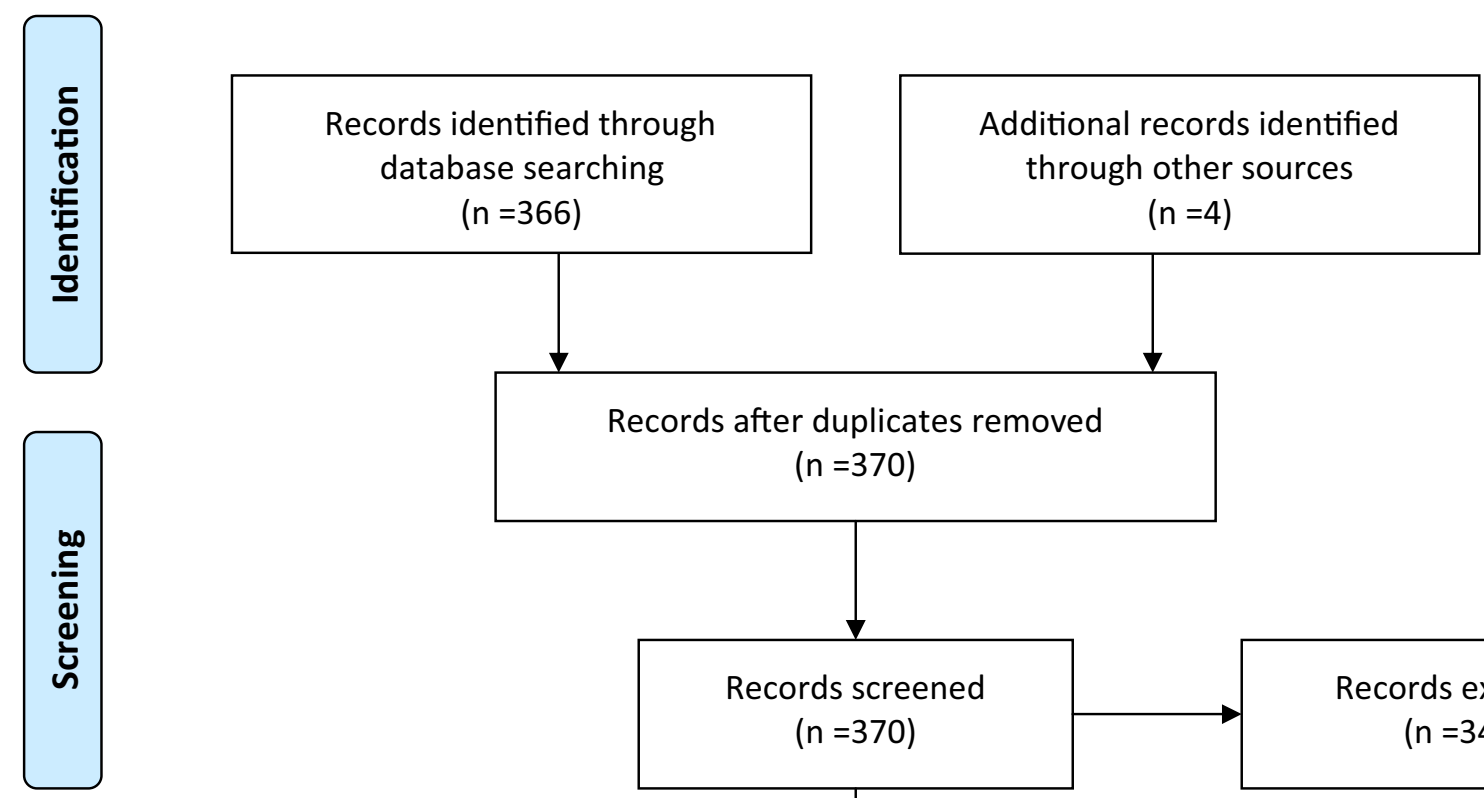

Records after duplicates removed

$$
\text { ( } n=370)
$$
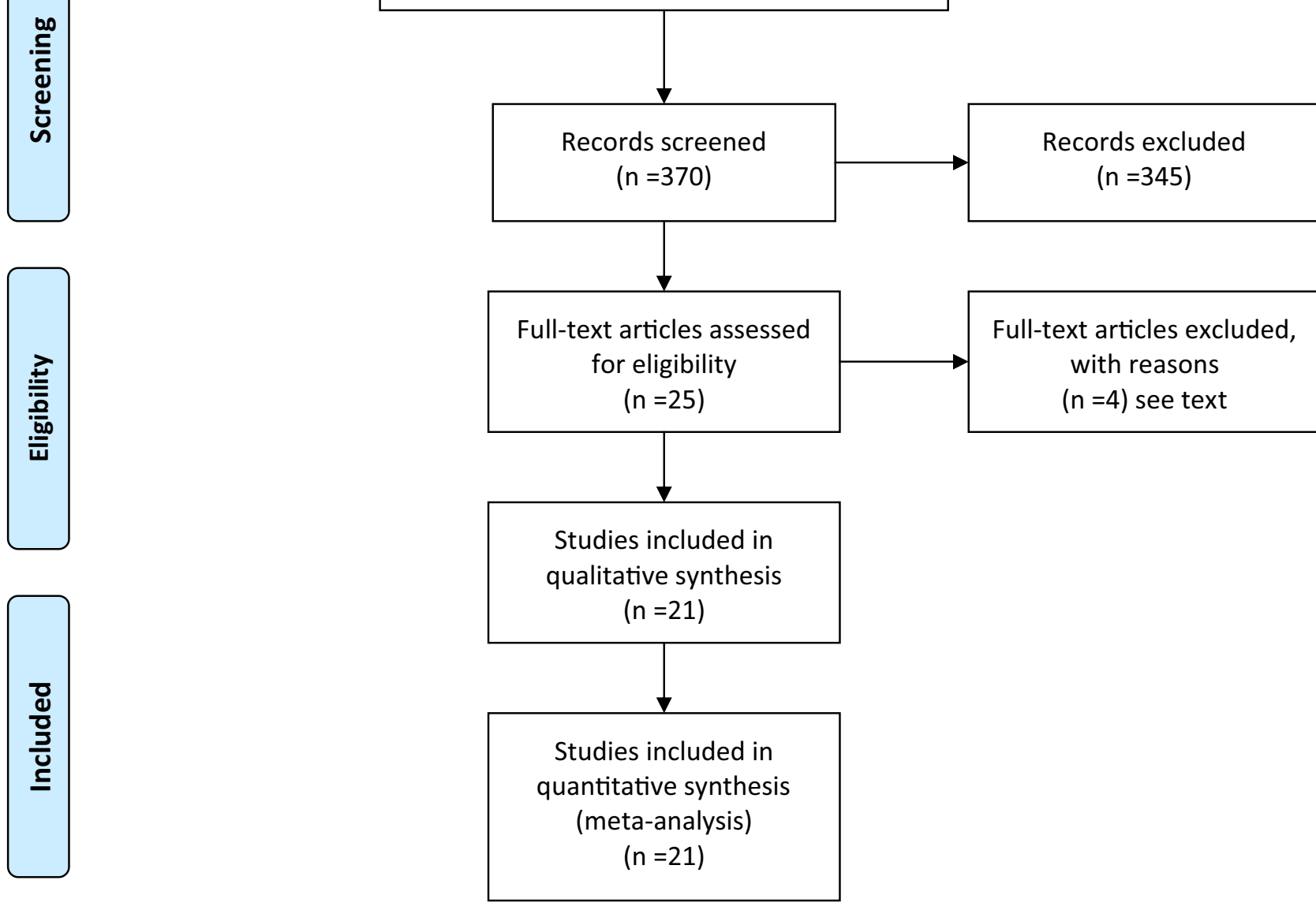

Fig. 1 Diagram of search strategy 
Table 1 Outcomes of Interest

\begin{tabular}{|c|c|c|c|c|}
\hline $\begin{array}{l}\text { Outcome of } \\
\text { Interest }\end{array}$ & $\begin{array}{l}\text { Number of studies } \\
\text { and patients } \\
\text { (\%; events/total) }\end{array}$ & $\begin{array}{l}\text { Statistical method, } \\
\text { estimated effect, } 95 \% \mathrm{CI}\end{array}$ & $p$-value & $\begin{array}{l}\mathrm{I}^{2} \\
(\%)\end{array}$ \\
\hline Age ${ }^{9-18,20,23-27,29}$ & 17,5458 & $\mathrm{MD}=-87(-2.13,0.39)$ & .09 & 61 \\
\hline $\begin{array}{l}\text { Operative Time } \\
9-15,18,19,22,26,29\end{array}$ & 11,1555 & $\mathrm{MD}=11.05(1.68,20.42)$ & .002 & 98 \\
\hline Haematoma $_{11,14,15,20,26,29}^{9-}$ & $\begin{array}{l}9,1255 \\
(2.9 \% ; 12 / 420) \\
(12 \% ; 56 / 463)\end{array}$ & P. $\mathrm{OR}=0.26(0.16,0.41)$ & $\therefore .001$ & 23 \\
\hline $\begin{array}{l}\text { Seroma } \\
9-12,14,15,18,20,21,26\end{array}$ & $\begin{array}{l}10,1201 \\
(5 \% ; 29 / 570) \\
(4.6 \% ; 29 / 631)\end{array}$ & P. $O R=1.05(0.62,1.80)$ & .50 & 50 \\
\hline $\begin{array}{l}\text { Wound Infections } \\
9,11-16,18-20,22,29\end{array}$ & $\begin{array}{l}12,3552 \\
(1 \% ; 17 / 1562) \\
(2 \% ; 33 / 1618)\end{array}$ & P. OR=0.52(0.30, 0.89) & .02 & 0 \\
\hline$\underset{10-12,16,19}{\text { Vascular Injury }}$ & $\begin{array}{l}5,2366 \\
(1.3 \% ; 15 / 1173 \\
(0.5 \% ; 6 / 1193)\end{array}$ & P. $O R=2.49(1.05,5.88)$ & .04 & 55 \\
\hline $\begin{array}{l}\text { Return to Usual } \\
\text { activities }^{9-15,19,29}\end{array}$ & 8,1286 & $\mathrm{MD}=-6.32(-8.17,-4.48)$ & $n \cap 1$ & 70 \\
\hline $\begin{array}{l}\text { Time to } \\
\text { Discharge } \\
10,12,1418,19\end{array}$ & 5,514 & $\mathrm{MD}=-0.09(-1.99,1.82)$ & .93 & 93 \\
\hline $\begin{array}{l}\text { Recurrences } \\
12,13,15-17,19--25,27,29\end{array}$ & $\begin{array}{l}14,5468 \\
(6 \% ; 149 / 2678) \\
(4 \% ; 99 / 2790)\end{array}$ & P. OR=1.58(1.22, 2.04) & .005 & 63 \\
\hline$\underset{9-16,18-21,29}{\text { Persistent pain }}$ & $\begin{array}{l}13,3650 \\
(11 \% ; 178 / 1617) \\
(13 \% ; 219 / 1680)\end{array}$ & P. $\mathrm{OR}=0.81(0.66,1.00)$ & .05 & 38 \\
\hline $\begin{array}{l}\text { Impaired } \\
\text { Sensibility } \\
\text { S1,29 }^{9,10,12,19-}\end{array}$ & $\begin{array}{l}8,1144 \\
(6.4 \% ; 23 / 359) \\
(21 \% ; 86 / 413)\end{array}$ & P. OR=0.26(0.17, 0.40) & 6.00 & 0 \\
\hline
\end{tabular}

$P$. $O R$ Peto odds ratio, $M D$ mean difference, $C I$ confidence intervals

Green highlighted favours Lichtenstein; red highlighted favours TEP; $I^{2}$ : heterogeneity metric

the reoperation rates following TEP laparoscopic hernioplasty and Lichtenstein hernioplasty were $3.3 \%$ and $2.4 \%$, respectively [32]. It therefore suggests that the recurrence rates were around $6.6 \%$ and $4.8 \%$ for the laparoscopic and Lichtenstein hernioplasties, respectively. It is noteworthy that our study recorded recurrence rates of $6 \%$ and $4 \%$ for the TEP and Lichtenstein hernioplasties, respectively, which are similar to those of the above-mentioned study.

The reported incidence of clinically significant chronic persistent pain was $10-12 \%$ with a tendency to decrease with time [33, 34]. In this study, $11 \%$ of patients in the TEP cohort and $13 \%$ of the patients in the Lichtenstein 
Table 2 Risk of bias of RCTs

\begin{tabular}{|c|c|c|c|c|c|c|}
\hline Author & $\begin{array}{l}\text { Random } \\
\text { sequence gen- } \\
\text { eration }\end{array}$ & $\begin{array}{l}\text { Allocation } \\
\text { concealment }\end{array}$ & $\begin{array}{l}\text { Blinding of partici- } \\
\text { pants and personnel }\end{array}$ & $\begin{array}{l}\text { Blinding of out- } \\
\text { come assessment }\end{array}$ & $\begin{array}{l}\text { Incomplete } \\
\text { outcome data }\end{array}$ & Selective reporting \\
\hline Wright & Low & Low & High & High & High & Unclear \\
\hline Heikkinen et al. (1998) [10] & Low & Low & Unclear & High & Low & Low \\
\hline Gokalp & Low & High & High & High & Low & Low \\
\hline Andersson & Low & Low & High & High & Low & Low \\
\hline Colak & Low & Low & High & High & Unclear & Low \\
\hline Lal P & Unclear & Low & Unclear & Unclear & Unclear & High \\
\hline Bringman & Low & Low & Unclear & High & Low & Low \\
\hline Neumayer & Low & Unclear & Unclear & High & Low & Low \\
\hline Heikkinen et al. (2004) [17] & Low & Low & Unclear & High & Low & Low \\
\hline Lau & Unclear & High & Unclear & High & Low & High \\
\hline Dedemadi & Low & Low & Low & Unclear & Low & Low \\
\hline Pokorny & Low & Low & Low & Unclear & Low & Low \\
\hline Hallén & High & High & High & Unclear & Low & Low \\
\hline Eklund & Low & Low & High & High & Low & Low \\
\hline Kuhia & Low & Low & Low & Unclear & Low & Low \\
\hline Hamza & Low & High & High & High & Low & Low \\
\hline Eker & Low & Low & Unclear & Unclear & Low & Low \\
\hline Dhankhar & Low & Low & High & High & Low & Low \\
\hline Wang & High & High & High & High & Unclear & Unclear \\
\hline Moreno-Egea & Unclear & Low & Unclear & High & Low & Low \\
\hline Gutlic & Low & Low & Low & Low & Low & Low \\
\hline Pooled estimates & $\begin{array}{l}\text { Low-risk } \\
16 \text { studies }\end{array}$ & $\begin{array}{l}\text { Low-risk } \\
15 \text { studies }\end{array}$ & $\begin{array}{l}\text { Low-risk } \\
4 \text { studies }\end{array}$ & $\begin{array}{l}\text { Low-risk } \\
1 \text { study }\end{array}$ & $\begin{array}{l}\text { Low-risk } \\
17 \text { studies }\end{array}$ & $\begin{array}{l}\text { Low-risk } \\
17 \text { studies }\end{array}$ \\
\hline
\end{tabular}

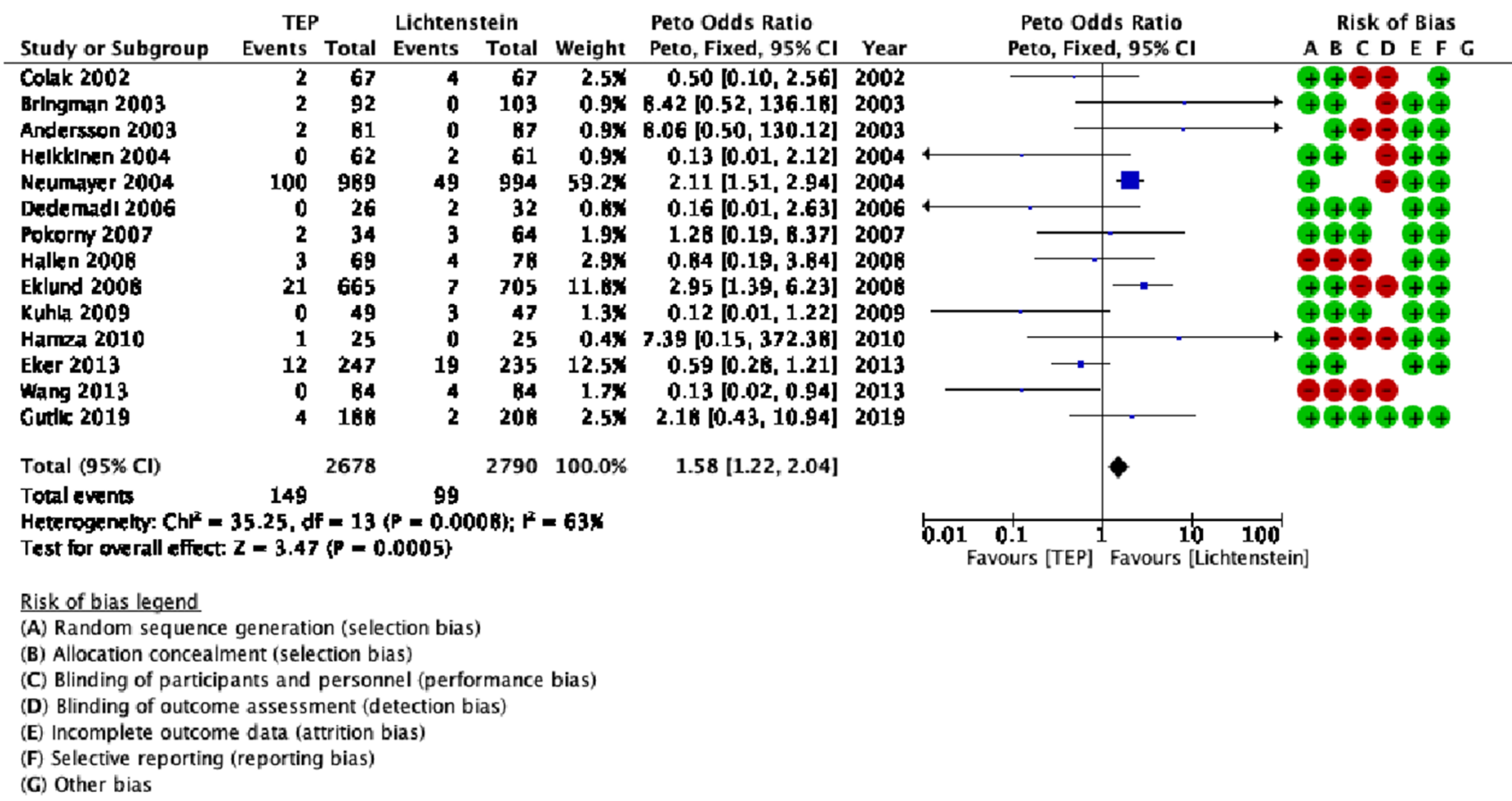

Fig. 2 Forest plot of recurrences 
(A) Traditional MA Recurrences TEP vs Lichtenstein

\begin{tabular}{|c|c|c|c|c|c|c|c|c|c|}
\hline Study or Subgroup & \multicolumn{2}{|l|}{ TEP } & \multicolumn{2}{|c|}{ Lichtenstein } & Weight & \multicolumn{2}{|l|}{ Peto Odds Ratio } & \multicolumn{2}{|c|}{$\begin{array}{l}\text { Peto Odds Ratio } \\
\text { Peto, Fixed, } 95 \% \mathrm{CI}\end{array}$} \\
\hline Colak 2002 & 2 & 67 & 4 & 67 & $2.5 \%$ & $0.50[0.10,2.56]$ & 2002 & & \\
\hline Bringman 2003 & 2 & 92 & 0 & 103 & $0.9 \times$ & $8.42[0.52,136.18]$ & 2003 & & \\
\hline Andersson 2003 & 2 & 81 & 0 & B7 & $0.9 \times$ & $8.06[0.50,130.12]$ & 2003 & & \\
\hline Helkkinen 2004 & D & 62 & 2 & 61 & $0.9 \%$ & $0.13[0.01,2.12]$ & 2004 & $\leftarrow$ & \\
\hline Neumayer 2004 & 100 & 989 & 49 & 994 & $59.2 \%$ & $2.11[1.51,2.94]$ & 2004 & & 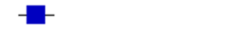 \\
\hline Dedemadl 2006 & 0 & 26 & 2 & 32 & D. $\mathbf{B} \mathbf{3}$ & $0.16[0.01,2.63]$ & 2006 & 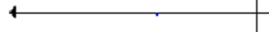 & \\
\hline Popkorny 2007 & 2 & 34 & 3 & 64 & $1.9 x$ & $1.28[0.19,8.37]$ & 2007 & & \\
\hline Hallen zouos & 3 & 69 & 4 & 78 & $2.9 \times$ & $0.64[0.19,3.14]$ & 2008 & & \\
\hline Eklund 2008 & 21 & 665 & 7 & 705 & 11. Bx & $2.95[1.39,6.23]$ & 2008 & & \\
\hline Kuhla 2009 & 0 & 49 & 3 & 47 & $1.3 \%$ & $0.12[0.01,1.22]$ & 2009 & & \\
\hline Hamza 2010 & 1 & 25 & 0 & 25 & $0.4 \%$ & $7.39[0.15,372.38]$ & 2010 & & $\longrightarrow$ \\
\hline Eker 2013 & 12 & 247 & 19 & 235 & $12.5 \%$ & $0.59[0.2 B, 1.21]$ & 2013 & & \\
\hline Wang 2013 & 0 & 84 & 4 & B4 & $1.7 \%$ & $0.13[0.02,0.94]$ & 2013 & & \\
\hline Gutik 2019 & 4 & $18 \mathrm{~B}$ & 2 & $20 B$ & $2.5 \%$ & $2.18[0.43,10.94]$ & 2019 & & \\
\hline Total $(95 \% \mathrm{CI})$ & & 2678 & & 2790 & $100.0 \%$ & $1.58[1.22,2.04]$ & & & \\
\hline Total events & 149 & & 99 & & & & & & \\
\hline $\begin{array}{l}\text { Heterogenelty: Chr" } \\
\text { Test for overall effe }\end{array}$ & $\begin{array}{l}.25, \mathrm{df} \\
=3.47\end{array}$ & $=13$ & $=0.0$ & a) 12 & 637 & & & $\begin{array}{l}0.1 \\
\text { Favours [TEP] }\end{array}$ & $\begin{array}{cc}10 & 100 \\
\text { Favours [Lichtenstein] }\end{array}$ \\
\hline
\end{tabular}

(B) Cumulative Meta-Analysis: Recurrences

Study

ID

Oddsratio $(95 \% \mathrm{Cl})$

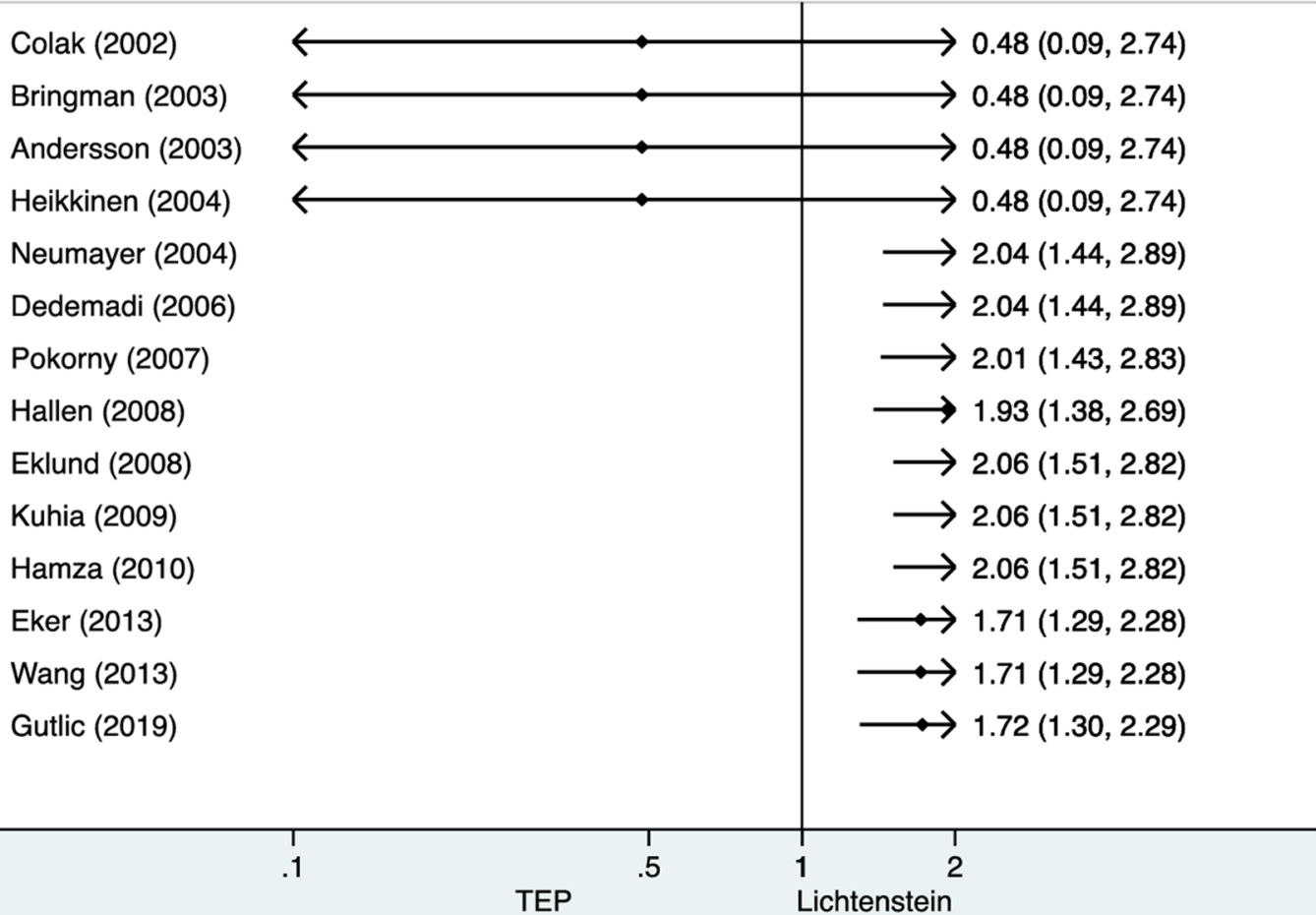

Fig. 3 a Traditional meta-analysis of recurrences; $\mathbf{b}$ cumulative meta-analysis of recurrences 


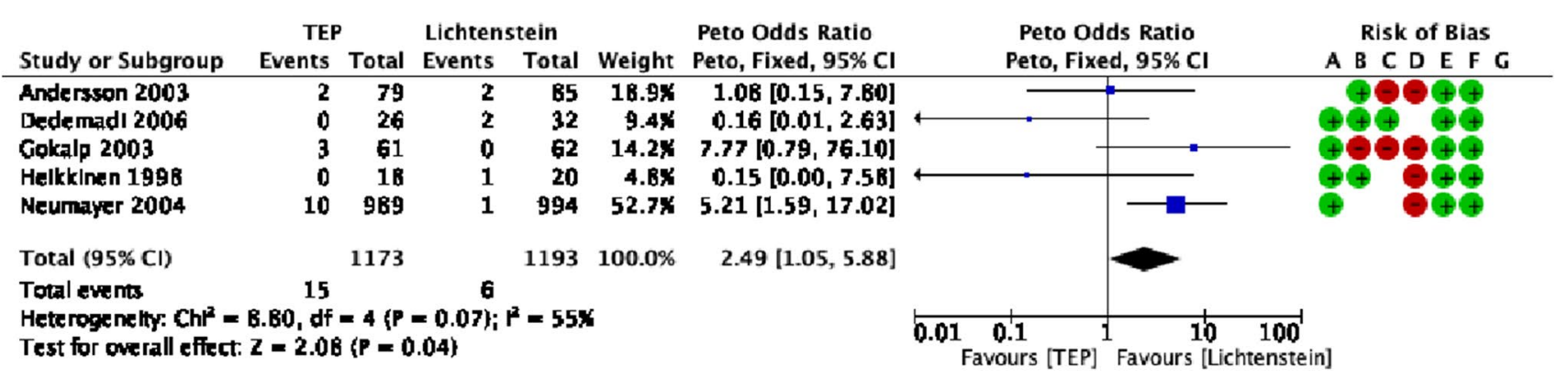

Risk of bias legend
(A) Random sequence generation (selection bias)
(B) Allocation concealment (selection bias)
(C) Blinding of participants and personnel (performance bias)
(D) Blind ing of outcome assessment (detection bias)
(E) Incomplete outcome data (attrition bias)
(F) Selective reporting (reporting bias)
(G) Other bias

Fig. 4 Forest plot of vascular injuries

cohort had chronic persistent pain, but no statistically significant differences were observed between the cohorts.

The reported incidence of vascular injury during inguinal hernioplasty is $0.1-0.4 \%$ [35]. Data from the German registry Herniamed reported significantly more vascular injuries of $1.39 \%$ in TEP compared to $1.13 \%$ in TAPP [36]. In this study, the rate of vascular injuries was significantly higher in the TEP cohort at $1.3 \%$ compared to $0.5 \%$ in the Lichtenstein cohort (Fig. 4, Table 1).

Furthermore, the haematoma formation rate was significantly less in the TEP cohort than in the open cohort. However, the lack of a haematoma severity classification and a common haematoma definition that is clinically relevant for the laparoscopic and open approaches make the extrapolation of objective conclusions difficult. Other potential contributors to diagnostic bias are preperitoneal haematomas that may be of similar sizes to superficial haematomas of open procedures, but may not be as easily diagnosable as those of the open procedure [1].

Cumulative meta-analysis further supports the findings of traditional meta-analysis by demonstrating that from 2004 until present, the recurrence rate is significantly lower for Lichtenstein repair. Interestingly, the most recently published high-quality RCT by Gutlic [29] did not influence the accumulated evidence.

To the best of the authors' knowledge, this is the most up-to-date study and the first cumulative meta-analysis with 21 included studies and 6573 enrolled patients compared to the previous meta-analysis with 14 studies and 3279 patients [3]. However, the results of this study should be interpreted with caution due to the study limitations. The overall quality of the included RCTs was poor; only 4 of the 21 studies blinded the participants and the personnel, and one of them blinded the outcome assessors (Table 2). The total sample was quite heterogeneous as it included patients with primary, recurrent, and bilateral hernias. In addition, the studies were conducted in single centres and the follow-up periods varied widely (Table 3). Therefore, national and institutional characteristics, underpowered and heterogeneous samples, performance, and detection bias may have influenced the results. However, the outcome measures described in this study provide contemporaneous comparative data to allow surgeons to discuss the potential risks, benefits, and alternative treatment options with patients considering their options for inguinal hernia repair.

\section{Implications for research}

To shed further light on the topic, multicentre RCTs with the following characteristics should be conducted: strict adherence to standards recommended in the Consolidated Standards of Reporting Trials (CONSORT) guidelines; comparison of primary, recurrent, and simultaneously performed bilateral hernias in separate patient groups; adequate sample power with predefined outcome measures critical for decision making according to the Grading of Recommendations Assessment, Development, and Evaluation system (GRADE); blind outcome assessors (although we acknowledge that this would be very challenging when assessing longer term outcomes); common methods of outcome assessment; and a follow-up period at least 3 years $[37,38]$. 
Table 3 Study characteristics

\begin{tabular}{|c|c|c|c|}
\hline Author (year) & $\begin{array}{l}\text { Number of patients } \\
\text { TEP-L }\end{array}$ & Age TEP-L & $\begin{array}{l}\text { FU period } \\
\text { (months) }\end{array}$ \\
\hline Wright et al. (1995) [9] & $60-60$ & $\begin{array}{l}\quad 63 \pm 6.25 \\
68 \pm 6.5\end{array}$ & NR \\
\hline Heikkinen et al. (1998) [10] & $18-20$ & $\begin{array}{l}51 \pm 8.5 \\
55.5 \pm 10\end{array}$ & NR \\
\hline Gokalp et al. (2003) [11] & $61-62$ & $\begin{array}{l}47 \pm 10.75 \\
45 \pm 10.5\end{array}$ & 18 \\
\hline Andersson et al. (2003) [12] P\&R & $81-87$ & $\begin{array}{l}50 \pm 9 \\
49 \pm 9\end{array}$ & 12 \\
\hline Colak et al. (2003) [13] & $67-67$ & $\begin{array}{l}49.4 \pm 14.25 \\
51.6 \pm 15.25\end{array}$ & 12 \\
\hline Lal et al. (2003) [14] & $25-25$ & $\begin{array}{l}36.72 \pm 12.08 \\
37.8 \pm 12.43\end{array}$ & 13 \\
\hline Bringman et al. (2003) [15] & $92-103$ & $\begin{array}{l}55 \pm 12 \\
54 \pm 11\end{array}$ & 20 \\
\hline Neumayer et al. (2004) [16] & 989-994 & $\begin{array}{l}58.6 \pm 12.8 \\
58.4 \pm 12.7\end{array}$ & 24 \\
\hline Heikkinen et al. (2004) [17] & $62-61$ & $\begin{array}{l}46 \pm 12 \\
48 \pm 13\end{array}$ & 60 \\
\hline Lau et al. (2006) [18] & $100-100$ & $\begin{array}{l}55 \pm 15.5 \\
56 \pm 13.1 \\
p=0.583\end{array}$ & 12 \\
\hline Dedemadi et al. (2006) [19] & $26-32$ & NR & 36 \\
\hline Pokorny et al. (2007) [20] & $36-69$ & $\begin{aligned} & 48 \pm 13.5 \\
52 & \pm 16.25\end{aligned}$ & 36 \\
\hline Hallén et al. (2008) [21] & $92-93$ & NR & 87 \\
\hline Eklund et al. (2008) [23] & $665-705$ & $\begin{array}{l}53 \pm 9.6 \\
52 \pm 10.1\end{array}$ & 60 \\
\hline Kuhia et al. (2009) [24] & $49-47$ & $\begin{array}{l}57.8 \pm 12.6 \\
55.8 \pm 12\end{array}$ & 60 \\
\hline Hamza et al. (2010) [22] & $25-25$ & $\begin{array}{l}34.91 \pm 13 \\
35.12 \pm 10.1\end{array}$ & 6 \\
\hline Eker et al. (2012) [25] & $336-324$ & 5556 & 60 \\
\hline Dhankhar et al. (2013) [26] & $29-30$ & $\begin{array}{c}38.17 \pm 11.53 \\
43.20 \pm 13.59\end{array}$ & 3 \\
\hline Wang et al. (2013) [27] & $84-84$ & $\begin{array}{l}48.25 \pm 17.09 \\
52.12 \pm 17.46\end{array}$ & 16 \\
\hline Moreno-Egea et al. (2014) [28] & $106-102$ & NR & 24 \\
\hline Gutlic et al. (2019) [29] & $239-241$ & $\begin{array}{l}51 \pm 12 \\
54 \pm 12\end{array}$ & 36 \\
\hline Pooled estimates & $\begin{array}{l}3242-3331 \\
\text { total } 6573\end{array}$ & $\begin{array}{c}\mathrm{OR}=-1.09(-2.33,0.15) \\
\quad p=0.09\end{array}$ & \\
\hline
\end{tabular}

TEP total extraperitoneal, $L$ Lichtenstein, $F U$ follow-up

Funding None.

\section{Compliance with ethical standards}

Conflict of interest All authors declare they have no conflicts of interest or financial ties to disclosure.

Ethical approval This article did not require ethical approval of any kind.
Human and animal rights This study does not contain any studies with human participants or animals performed by any of the authors.

Informed consent For this type of study, formal consent was not necessary.

Open Access This article is distributed under the terms of the Creative Commons Attribution 4.0 International License (http://creativeco mmons.org/licenses/by/4.0/), which permits unrestricted use, distribution, and reproduction in any medium, provided you give appropriate 
credit to the original author(s) and the source, provide a link to the Creative Commons license, and indicate if changes were made.

\section{References}

1. The Hernia Surge Group (2018) International guidelines for groin hernia management. Hernia 22:1-165

2. Koning GG, Wetterslev J, van Laarhoven CJHM et al (2013) The totally extraperitoneal method versus Lichtenstein's technique for inguinal hernia repair: a systematic review with metaanalyses and trial sequential analyses of randomized clinical trials. PLOS 8:e52599

3. Bobo Z, Nan W, Qin Q et al (2014) Meta-analysis of randomized controlled trials comparing Lichtenstein and totally extraperitoneal laparoscopic hernioplasty in treatment of inguinal hernias. J Surg Res 192:409-420

4. Moher D, Liberati A, Tetzlaff J, Altman DG (2009) Preferred reporting items for systematic reviews and meta-analyses: the PRISMA statement. PLoS Med 6(6):e1000097. https://doi. org/10.1371/journal.pmed.1000097

5. Higgins JPT, Greens S (2011) Cochrane handbook for systematic reviews of interventions. Willey, Chichester (The Cochrane library issue 4)

6. Higgins JP, Thompson SG, Deeks JJ, Altman DG (2003) Measuring inconsistency in meta-analyses. BMJ 327:557-560

7. Hozo SP, Diulbegovic B, Hozo I (2005) Estimating the mean and variance from the median, range, and the size of a sample. BMC Med Res Methodol 5:13

8. Sterne JA (1998) Cumulative meta-analysis. Stata Tech Bull 42:13-16

9. Wright DM, Kennedy A, Baxter JN, Fullarton GM, Fife LM, Sunderland GT, O'Dwyer PJ (1996) Early outcome after open versus extraperitoneal endoscopic tension-free hernioplasty: A randomised trial. Surgery 119:552-557

10. Heikinen T, Haukipuro K, Hulko A (1998) A cost and outcome comparison between laparoscopic and Lichtenstein hernia operations in a day-case unit. Surg Endosc 12:1199-1203

11. Gokalp A, Inal M, Maralcan G, Baskonus I (2003) A prospective randomized study of Lichtenstein open tension-free versus laparoscopic totally extraperitoneal techniques for inguinal hernia repair. Acta Chir Belg 103:502-506

12. Andersson B, Hallén M, Leveau P, Bergenfelz A, Westerdahl J (2003) Laparoscopic extraperitoneal inguinal hernia repair versus open mesh repair: a prospective randomized controlled trial. Surgery 133:464-472

13. Colak T, Akca T, Kanik A, Aydin S (2003) Randomized clinical trial comparing laparoscopic totally extraperitoneal approach with open mesh repair in inguinal hernia. Surg Laparoscop Endosc Percutan Tech 13:191-195

14. Lal P, Kajla RK, Chander J, Saha R, Ramteke VK (2003) Randomized controlled study of laparoscopic total extraperitoneal versus open Lichtenstein inguinal repair. Surg Endosc 17:850-856

15. Bringman S, Ramel S, Heikkinen TJ, Englund T, Westman B, Anderberg B (2003) Tension-free inguinal hernia repair: tEP versus Mesh-plug versus Lichtenstein. A prospective randomized trial. Ann Surg 237:142-147

16. Neumayer L, Giobbie-Hurder A, Jonasson O, Fitzgibbons R, Dunlop D, Gibbs J, Reda D, Hendersen W (2004) Open mesh versus laparoscopic mesh repair of inguinal hernia. N Engl J Med 350:1819-1827

17. Heikkinen T, Bringman S, Ohtonen P, Kunelius P, Haukipuro K, Hulkko A (2004) Five -year outcome of laparoscopic and Lichtenstein hernioplasties. Surg Endosc 18:518-522
18. Lau H, Patil NG, Yuen WK (2006) Day-case endoscopic totally extraperitoneal inguinal hernioplasty versus open Lichtenstein hernioplasty for unilateral primary inguinal hernia in males. A randomized trial. Surg Endosc 20:76-81

19. Dedemadi G, Sgourakis J, Karaliotas C, Christofides T, Kouraklis G, Karaliotas C (2006) Comparison of laparoscopic and open tension-free repair of recurrent inguinal hernias: a prospective randomized study. Surg Endosc 20:1099-1104

20. Pokorny H, Klingler A, Schmid T, Fortely R, Hollinsky C, Kawji R, Steiner E, Pernthaler H, Függer R, Scheyer M (2008) Recurrence and complications after laparoscopic versus open inguinal hernia repair: results of a prospective randomized multicentre trial. Hernia 12:385-389

21. Hallén M, Bergenfelz A, Westerdahl J (2008) Laparoscopic extraperitoneal inguinal hernia repair versus open mesh repair: long-term follow-up of randomized controlled trial. Surgery 143:313-317

22. Hamza Y, Gabr E, Hammadi H, Khalil R (2010) Four-arm randomized trial comparing laparoscopic and open hernia repairs. Int J Surg 8:25-28

23. Eklund AS, Montgomery AK, Rasmussen C, Sandbue RP, Bergkvist LA, Rudberg CR (2009) Low recurrence rate after laparoscopic (TEP) and open (Lichtenstein) inguinal hernia repair. A randomized multicentre trial with 5-year follow-up. Ann Surg 249:33-38

24. Kuhia ST, Huttunen R, Silvasti SO, Heiskanen JT, Ahtola H, Uotila-Nieminen M et al (2009) Lichtenstein hernioplasty versus totally extraperitoneal laparoscopic hernioplasty in treatment of recurrent inguinal hernia- A prospective randomized trial. Ann Surg 249:384-387

25. Eker HH, Langeveld HR, Klitsie PJ, van't Riet M, Stassen LP, Weidema WF, Steyerberg EW, Lange JF, Bonjer HJ, Jeekel J (2012) Randomized clinical trial of total extraperitoneal inguinal hernioplasty vs Lichtenstein repair: a long-term follow-up study. Arch Surg. 147:256-260

26. Dhankhar DS, Sharma N, Mishra T, Kaur N, Singh S, Gupta S (2014) Totally extraperitoneal repair under general anesthesia versus Lichtenstein repair under local anesthesia for unilateral inguinal hernia: a prospective randomized controlled trial. Surg Endosc 28:996-1002

27. Wang WJ, Chen JZ, Fang Q, Li JF, Jin PF, Li ZT (2013) Comparison of the effects of the laparoscopic hernia repair and Lichtenstein tension-free hernia repair. J Laparoendosc Adv Surg Tech 23:301-305

28. Moreno-Egea A (2014) Is it possible to eliminate sutures in open (Lichtenstein technique) and laparoscopic (totally extraperitoneal endoscopic) inguinal hernia repair? A randomized controlled trial with tissue adhesive ( $n$-hexyl-a-cyanoacrylate). Surg Innov 21:590-599

29. Gutlic N, Gutlic A, Peterson U, Rogmark P, Montogomery A (2019) Ranodomized clinical trial comparing total extraperitoneal with Lichtenstein inguinal repair (TEPLICH trial). BJS 106:845-855

30. Staarink M, Van Veen RN, Hop WC, Weidema WF (2008) A 10-year repair follow-up study on endoscopic total extraperitoneal repair of primary and recurrent inguinal hernia. Surg Endosc 22:1803-1806

31. Sevonius D, Gunnarsson U, Nodin P, Nilsson E, Sandblom G (2011) Recurrent groin hernia surgery. Br J Surg 98:1489-1494

32. Burcharth J, Andresen K, Pommergaard H-C, Bisgaard T, Rosenberg J (2014) Recurrence patterns of direct and indirect inguinal hernias in a nationwide population in Denmark. Surgery 155:173-177

33. Alfieri S, Amid PK, Campanelli G, Izard G, Kehlet H, Wijsmuller AR, Di Miceli D, Doglietto GB (2011) International guidelines for prevention and management of post-operative chronic pain following inguinal hernia surgery. Hernia 15:239-249 
34. Nienhuijs SW, Rosman C, Strobbe LJA, Wolff A, Bleichrodt RP (2008) An overview of the features influencing pain after inguinal hernia repair. Int J Surg 6:351-356

35. Spaw AT, Ennis BW, Spaw LP (1991) Laparoscopic hernia repair: the anatomic basis. J Laparosc Surg 1:269-277

36. Köckerling F, Bittner R, Jacob DA, Schug-Pass C, Laurenz C, Adolf D, Keller T, Stechemesser B (2015) TEP versus TAPP: comparison of the perioperative outcome in 17587 patients with primary unilateral inguinal hernia. Surg Endosc 29:3750-3760

37. Schulz KF, Altman DE, Moher D, CONSORT Group, CONSORT 2010 (2010) Updated guidelines for reporting parallel group randomized trials. PLos Med. 7:e1000251
38. Gyatt GH, Oxman AD, Kunz R, Vist GE, Falck-Ytter Y, Schünemann HJ (2008) GRADE working group: what is "quality of evidence" and why is it important to clinicians? BMJ 336:995-998

Publisher's Note Springer Nature remains neutral with regard to jurisdictional claims in published maps and institutional affiliations. 